Article

\title{
Sustained-Release Microspheres of Rivoceranib for the Treatment of Subfoveal Choroidal Neovascularization
}

\author{
E Seul Kim ${ }^{1,+}$, Min Sang Lee ${ }^{1,+}$, Hayoung Jeong ${ }^{2,3}$, Su Yeon Lim ${ }^{1}$, Doha Kim ${ }^{2,3}$, Dahwun Kim ${ }^{1}$, Jaeback Jung ${ }^{1}$, \\ Siyan Lyu ${ }^{1}$, Hee Joo Cho ${ }^{1}$, Dong Min Kim ${ }^{1}$, Wonhee Suh ${ }^{2,3, * \mathbb{D}}$ and Ji Hoon Jeong ${ }^{1, *(D)}$
}

1 School of Pharmacy, Sungkyunkwan University, Suwon 16419, Korea; seul4146@skku.edu (E.S.K.); lminsa@skku.edu (M.S.L.); kally37@naver.com (S.Y.L.); gnjs0219@naver.com (D.K.); domonde@naver.com (J.J.); lsy2018dd@hotmail.com (S.L.); chohj823@naver.com (H.J.C.); eastjade@g.skku.edu (D.M.K.)

2 Department of Global Innovative Drug, Graduate School of Chung-Ang University, Seoul 06974, Korea; gkdud819@naver.com (H.J.); summerflow02@gmail.com (D.K.)

3 College of Pharmacy, Chung-Ang University, Seoul 06974, Korea

* Correspondence: wsuh@cau.ac.kr (W.S.); jhjeong@skku.edu (J.H.J.); Tel.: +82-02-820-5960 (W.S.); +82-31-290-7783 (J.H.J.)

+ These authors contributed equally to this work.

\section{check for} updates

Citation: Kim, E.S.; Lee, M.S.; Jeong, H.; Lim, S.Y.; Kim, D.; Kim, D.; Jung, J.; Lyu, S.; Cho, H.J.; Kim, D.M.; et al. Sustained-Release Microspheres of Rivoceranib for the Treatment of Subfoveal Choroidal Neovascularization. Pharmaceutics 2021, 13, 1548. https://doi.org/ $10.3390 /$ pharmaceutics 13101548

Academic Editor: Monica

M. Jablonski

Received: 18 August 2021

Accepted: 19 September 2021

Published: 24 September 2021

Publisher's Note: MDPI stays neutral with regard to jurisdictional claims in published maps and institutional affiliations.

Copyright: (c) 2021 by the authors. Licensee MDPI, Basel, Switzerland. This article is an open access article distributed under the terms and conditions of the Creative Commons Attribution (CC BY) license (https:/ / creativecommons.org/licenses/by/ $4.0 /)$.

\begin{abstract}
The wet type of age-related macular degeneration (AMD) accompanies the subfoveal choroidal neovascularization (CNV) caused by the abnormal extension or remodeling of blood vessels to the macula and retinal pigment epithelium (RPE). Vascular endothelial growth factor (VEGF) is known to play a crucial role in the pathogenesis of the disease. In this study, we tried to repurpose an investigational anticancer drug, rivoceranib, which is a selective inhibitor of VEGF receptor-2 (VEGFR2), and evaluate the therapeutic potential of the drug for the treatment of wet-type AMD in a laser-induced CNV mouse model using microsphere-based sustained drug release formulations. The PLGA-based rivoceranib microsphere can carry out a sustained delivery of rivoceranib for 50 days. When administered intravitreally, the sustained microsphere formulation of rivoceranib effectively inhibited the formation of subfoveal neovascular lesions in mice.
\end{abstract}

Keywords: rivoceranib; drug repositioning; microsphere; subfoveal choroidal neovascularization; macular degeneration

\section{Introduction}

The eye is a representative organ, exhibiting the fastest signs of aging, and visual problems are generally more noticeable than other age-related disorders [1]. Age-related macular degeneration (AMD) is a leading cause of irreversible visual loss in older adults [2]. The impaired vision and blindness associated with AMD are due to atrophic and neovascular complications [3]. Although the dry or non-neovascular type of AMD is more prevalent than wet or neovascular AMD, wet AMD is often involved with severely reduced vision acuity and vision loss which occurs when the outgrowth of blood vessels in the choroidal region extends underneath the foveal avascular zone (subfoveal choroidal neovascularization (CNV)) [4,5]. Vascular endothelial growth factor (VEGF) is one of the most critical factors for the vascular proliferation and migration of endothelial cells in the neovascularization process [6]. Therefore, blocking the VEGF signaling pathway to prevent CNV has been popularly employed for the treatment of wet AMD [7]. Monoclonal antibodies targeting VEGF receptors (VEGFR) such as bevacizumab and ranibizumab are frequently used for CNV due to their desirable effectiveness and low incidence of serious ocular and systemic adverse events [8]. However, the lack of a proper delivery system and the high cost of antibody therapy have remained a limitation [9].

Corticosteroids are also widely used for ocular disorders involving CNV and macular edema, owing to their anti-inflammatory and anti-angiogenic properties [10]. Intravitreal 
injectable implants containing corticosteroids such as dexamethasone and fluocinolone acetamide could achieve sustained drug release in vitreous space for an extended period. However, steroid therapy is often associated with adverse events, including cataract formation, ocular hypertension, and glaucoma [11-13].

Biodegradable microspheres have been used for the extended release of various active substances including small molecules, polypeptides, and nucleic acids, since they are injectable and biodegradable, so additional surgical procedures for implant removal are not required [14]. In addition, microspheres can be formulated with hydrophobic as well as hydrophilic drugs and deliver the active substances for a longer period compared to nanoparticles [15]. Microspheres containing a small molecular protein kinase $C$ inhibitor (PKC412, Novartis Pharma) and fasudil were developed for CNV and glaucoma, respectively [16,17]. A sustained-release microsphere for an anti-VEGF RNA aptamer was also designed for the treatment of AMD [18].

Rivoceranib is a selective receptor tyrosine kinase inhibitor targeting the intracellular ATP-binding site of VEGFR2. Since VEGFR2 plays a crucial role in VEGF-mediated endothelial cell proliferation, migration, and permeabilization [19], rivoceranib was developed for antiangiogenic cancer therapy, and is in clinical trials for metastatic gastric cancer and metastatic adenoid cystic carcinoma [20]. We hypothesized that rivoceranib, a selective inhibitor of VEGFR tyrosine kinase, could be repositioned as a candidate for an anti-VEGF therapy for CNV. In our previous studies, human albumin-polyethyleneglycol (HSA-PEG) conjugate-based nanoparticles containing rivoceranib effectively reduced retinal vascular leakage and corneal neovascularization by blocking VEGF/VEGFR2 signaling [21,22]. In this study, injectable microsphere dosage forms of rivoceranib were developed for extended drug release in the vitreous space. The potential therapeutic effect of the rivoceranib microspheres for wet AMD was evaluated in a laser-induced CNV animal model.

\section{Materials and Methods}

\subsection{Materials}

Rivoceranib was obtained from HLB Bio (Seoul, Korea). Poly(d,l-lactic-co-glycolic acid) (PLGA) copolymers (Resomer RG502 (50:50, Mw 7000-17,000), Resomer RG502H (50:50, Mw 7000-17,000) and Resomer RG503H (50:50, Mw 24,000-38,000)), polyvinyl alcohol (PVA), and Tween 20 were purchased from Sigma (St. Louis, MO, USA). Methylene chloride, acetonitrile, and methanol were of analytical grade and used without purification (J. T. Baker, Phillipsburg, NJ, USA). All the liquid solutions were sterilized by autoclave or filtration ( $0.45 \mu \mathrm{m}$ filter unit, Millipore, Billerica, MA, USA).

\subsection{Preparation and Characterization of Rivoceranib Microspheres}

Microspheres containing rivoceranib were fabricated using an oil-in-water emulsification method. PLGA $(360 \mathrm{mg})$ and rivoceranib $(40 \mathrm{mg})$ were dissolved in methylene chloride/methanol (9:1 v/v, $1 \mathrm{~mL}$ ). The solution was slowly added to $0.5 \%$ PVA solution $(w / v, 300 \mathrm{~mL})$ and emulsified with a high-speed dispersion homogenizer (HG-15D, Daihan Scientific, Seoul, Korea) at either 1500 or $2000 \mathrm{rpm}$ for $2 \mathrm{~min}$. The resulting microspheres were centrifuged at $2000 \mathrm{rpm}$ at $4{ }^{\circ} \mathrm{C}$ and the supernatant was removed. The microspheres were resuspended in cold deionized water and washed three times by the centrifugation method. The collected microspheres were freeze-dried at $-70{ }^{\circ} \mathrm{C}$ and stored at $-20^{\circ} \mathrm{C}$ until use. The drug loading and encapsulation efficiency of the microspheres were determined using a Waters 626 HPLC pump equipped with a UV detector (Waters, Milford, MA, USA) and C18 reversed-phase column ( $5 \mu \mathrm{m}$ particle size; Millipore, Billerica, MA, USA). The mobile phase (acetonitrile and $0.01 \mathrm{M} \mathrm{KH}_{2} \mathrm{PO}_{4}$ in deionized water, 70:30 v/v) was delivered at a flow rate of $1 \mathrm{~mL} / \mathrm{min}$. The detection wavelength was set at $260 \mathrm{~nm}$. The morphology and size distribution of the rivoceranib microspheres were observed using scanning electron microscopy (SEM, JSM7600F, JEOL, Tokyo, Japan) and dynamic light scattering (Mastersizer 2000, Malvern Panalytical, Malvern, UK). 


\subsection{Differential Scanning Calorimetric (DSC) and Fourier-Transform Infrared (FTIR) Spectral Studies}

A mixture of PLGA and rivoceranib $(1: 1, w / w)$ was dissolved in a cosolvent of methylene chloride/methanol $(9: 1, v / v)$ and the solvent was evaporated to form a film. For DSC analysis, one milligram of the film sample was placed in an aluminum pan and its thermal behavior was monitored by applying heat from $25^{\circ} \mathrm{C}$ to $200{ }^{\circ} \mathrm{C}$ at a heating rate of $5^{\circ} \mathrm{C} / \mathrm{min}$ (DSC 6100, Seiko Instruments, Chiba, Japan). For FTIR analysis, the spectral profile of PLGA/rivoceranib film was observed in the range of 400 to $4000 \mathrm{~cm}^{-1}$ using FTIR spectrophotometer (IFS66v-S, Bruker, Billerica, MA, USA).

\subsection{Drug Release Profiles of PLGA/Rivoceranib Microspheres}

The PLGA/rivoceranib microspheres ( $5 \mathrm{mg}$ ) were suspended in $1 \mathrm{~mL}$ of deionized water. The suspension was transferred to a dialysis bag (MWCO 5000, Specrum, Gardena, CA, USA) and drug release was carried out in a $10 \mathrm{~mL}$ release medium $(0.1 \%$ polysorbate 80 in PBS pH 7.4) at $37^{\circ} \mathrm{C}$. The samples were taken at a predetermined period and the release medium was replenished. The amount of released drug was analyzed using HPLC.

\subsection{Animals}

The animals were cared for in accordance with the Guide for the Care and Use of Laboratory Animals published by the United States National Institutes of Health. The protocols were also approved by the Institutional Animal Care and Use Committee (IACUC) of Chung-Ang University (IACUC number: 201800044, start date: 4 May 2018). Nine- to ten-week-old male C57BL/ 6 mice were purchased from Orient Co., Ltd. (Seoul, Korea). Mice were housed in microisolator cages on individually ventilated cage racks with ad libitum access to an autoclaved standard rodent diet (LabDiet 5008, Purina, St. Louis, MO, USA) and were kept under a 12:12 h light/dark cycle. Anesthesia was performed via an intraperitoneal injection of ketamine hydrochloride $(100 \mathrm{mg} / \mathrm{kg}$ body weight) and xylazine hydrochloride ( $6 \mathrm{mg} / \mathrm{kg}$ body weight). The pupils of the anesthetized mice were dilated with topical drops of $1 \%$ tropicamide (Santen, Osaka, Japan).

\subsection{Mouse Model of Laser-Induced Choroidal Neovascularization (CNV)}

Immediately after mice were anesthetized and their pupils dilated, experimental CNV lesions (four spots per eye) were created at the 3, 6, 9, and 12 o'clock positions of the posterior pole of the fundus at equal distances from the optic nerve head with the following parameters: $532 \mathrm{~nm}$ wavelength, $50 \mu \mathrm{m}$ diameter, $70 \mathrm{~ms}$ duration, and $220 \mathrm{~mW}$ intensity (Micron IV image-guided laser system, Phoenix Research Laboratories, Pleasanton, CA, USA). Only mice with cavitation bubbles, which indicated the disruption of Bruch's membrane, were included in the study. Immediately after CNV induction, the mice were given an intravitreal injection of rivoceranib-loaded PLGA microspheres $(10 \mu \mathrm{g}$ rivoceranib $/ 10 \%$ RG502H-2.0k in $1 \mu \mathrm{L}$ PBS), PLGA microspheres (10\% RG502H-2.0k in $1 \mu \mathrm{L}$ PBS), or PBS $(1 \mu \mathrm{L})$. Two weeks later, the mice were euthanized for further analysis.

\subsection{Quantification of Laser-Induced CNV}

The eyes were enucleated and fixed in $4 \%$ paraformaldehyde solution in PBS. The posterior eyecups comprising the retinal pigmental epithelium, choroid, and sclera were microdissected from the surrounding tissues and prepared as flat mounts. The flat mounts were then stained with Alexa Fluor ${ }^{\circledR}$ 594-conjugated Griffonia simplicifolia isolectin B4 (IB4; 1:100 dilution; Invitrogen, Carlsbad, CA, USA) overnight at $4{ }^{\circ} \mathrm{C}$. Images of CNV lesions were obtained using a fluorescence microscope (Olympus, Tokyo, Japan); the exposure and gain were kept constant for all samples. In each whole-mount image, the numbers of pixels in the CNV areas were measured using ImageJ software (National Institute of Health, Bethesda, MD, USA). 


\subsection{Statistical Analysis}

All data are expressed as the mean \pm standard error of mean (SEM) of indicated $n$ values. One-way analysis of variance was used to determine the significance of differences between groups. Data were analyzed using the Bonferroni correction for multiple comparisons. Differences were considered significant when $p<0.05$.

\section{Results}

\subsection{Preparation and Characterization of Rivoceranib Microspheres}

The rivoceranib microspheres were prepared using an oil-in-water emulsification process, in which the manufacturing parameters, including the molecular weight and terminal functional group of PLGA polymers, and the emulsifying speeds for the formation of the oil-in-water emulsions were varied to observe the effect of the parameters on the release behavior of rivoceranib. The formulation parameters of the microspheres are summarized in Table 1. The fabrication parameters such as polymer end group did not significantly affect the efficiency of drug loading, suggesting there may be no interactions between the polymer end groups and rivoceranib.

Table 1. Rivoceranib microsphere formulations.

\begin{tabular}{|c|c|c|c|c|c|}
\hline Sample & $\begin{array}{c}\text { Polymer } \\
\text { Molecular Weight } \\
(\mathrm{Mw})^{\mathrm{a}}\end{array}$ & $\begin{array}{l}\text { Polymer end } \\
\text { Group }\end{array}$ & $\begin{array}{c}\text { Emulsification } \\
\text { Rate (rpm) }\end{array}$ & $\begin{array}{l}\text { Drug Loading } \\
\text { Content (\%) }\end{array}$ & $\begin{array}{l}\text { Encapsulation } \\
\text { Efficiency (\%) }\end{array}$ \\
\hline $502-1.5 \mathrm{k}$ & $7000-17,000$ & Hydroxyl & 1500 & 7.94 & 79.4 \\
\hline $502-2.0 \mathrm{k}$ & $7000-17,000$ & Hydroxyl & 2000 & 7.38 & 73.8 \\
\hline $502 \mathrm{H}-1.5 \mathrm{k}$ & $7000-17,000$ & Carboxyl & 1500 & 8.25 & 82.5 \\
\hline $502 \mathrm{H}-2.0 \mathrm{k}$ & $7000-17,000$ & Carboxyl & 2000 & 7.89 & 78.9 \\
\hline $503 \mathrm{H}-1.5 \mathrm{k}$ & $24,000-38,000$ & Carboxyl & 1500 & 7.80 & 78.0 \\
\hline $503 \mathrm{H}-2.0 \mathrm{k}$ & $24,000-38,000$ & Carboxyl & 2000 & 8.77 & 87.7 \\
\hline
\end{tabular}

${ }^{\mathrm{a}}$ as described in the manufacturer's specification.

The rivoceranib microspheres showed a spherical morphology with a smooth surface, as observed in a scanning electron microscope (SEM) (Figure 1). The microspheres prepared at $2000 \mathrm{rpm}$ of the emulsifying speed were smaller in size than those prepared at $1500 \mathrm{rpm}$ (Figure 2). However, the viscosity of the polymer due to the difference in molecular weight seemed not to be significant.
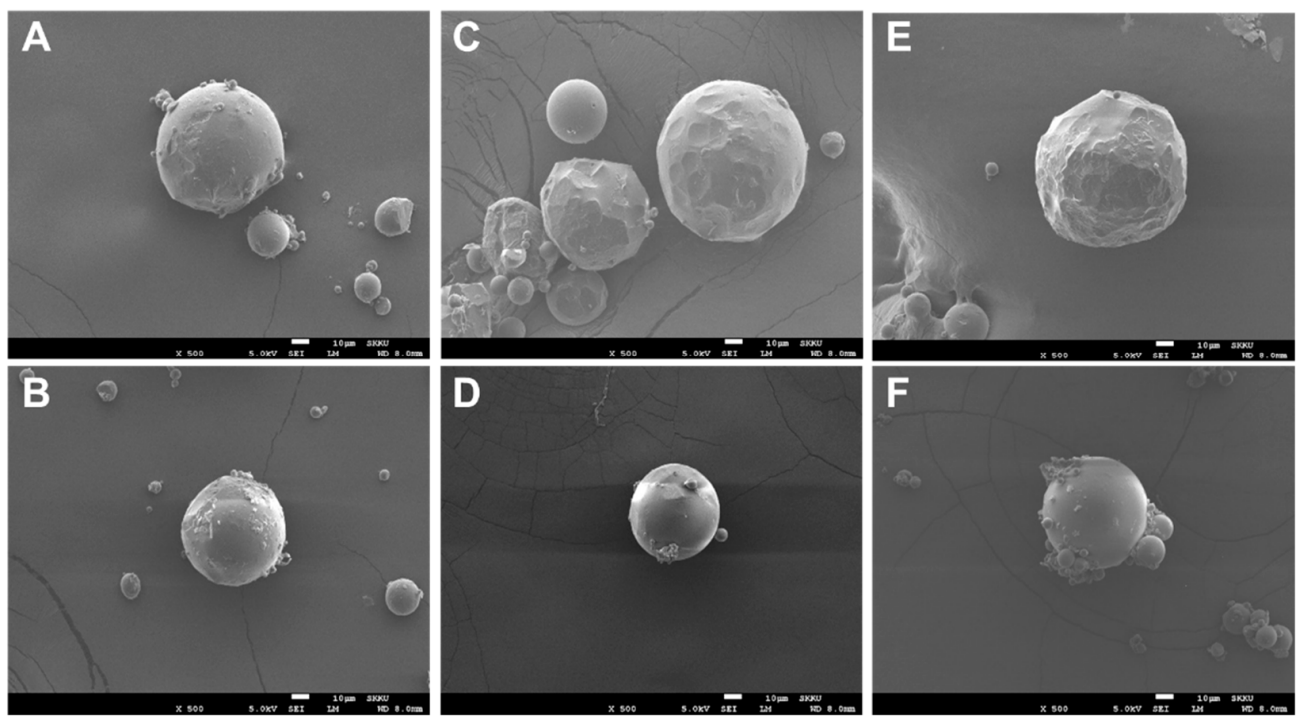

Figure 1. SEM images of rivoceranib microspheres. (A,B) microspheres were fabricated using PLGA RG502, (C,D) microspheres were from PLGA RG502H, (E,F) microspheres were from PLGA RG503H. Microspheres (A,C,E) were prepared at the emulsifying speed of $1500 \mathrm{rpm}$. Microspheres $(\mathbf{B}, \mathbf{D}, \mathbf{F})$ were prepared at $2000 \mathrm{rpm}$. Scale bars $=10 \mu \mathrm{m}$. 

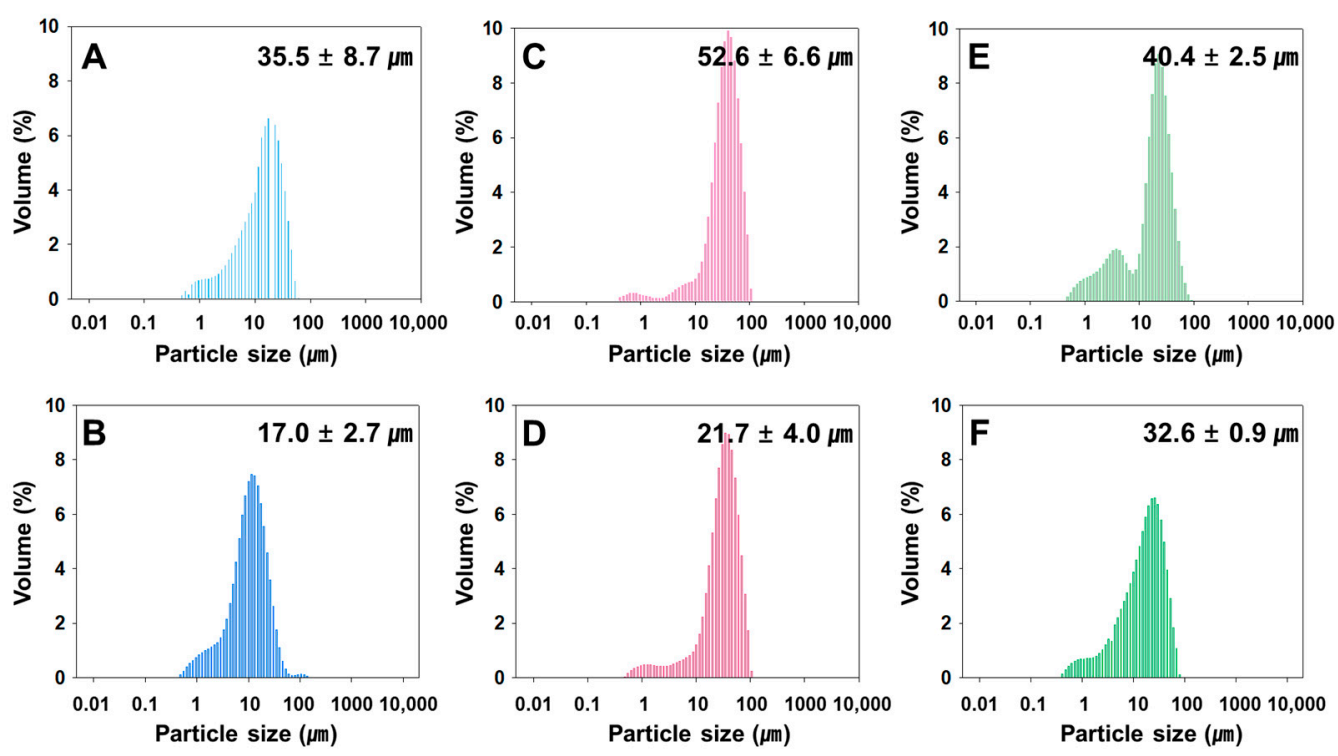

Figure 2. Size distribution of rivoceranib microspheres, determined by a light scattering method. (A,B) microspheres were fabricated using PLGA RG502, (C,D) microspheres were from PLGA RG502H, (E,F) microspheres were from PLGA RG503H. Microspheres (A,C,E) were prepared at the emulsifying speed of $1500 \mathrm{rpm}$. Microspheres $(\mathbf{B}, \mathbf{D}, \mathbf{F})$ were prepared at $2000 \mathrm{rpm}$.

\subsection{Polymer-Drug Interactions}

Interactions between a polymer matrix and a drug compound in a formulation are considered important since the interactions may affect the physicochemical properties and bioavailability of the drug [23]. The possibilities of polymer-drug interactions are often studied using the thermal and spectroscopic methods using DSC and FTIR, respectively [24]. The thermal behaviors of PLGA (RG502), rivoceranib, a physical mixture of PLGA and the drug $(1: 1, w / w)$, and polymer-drug blend films $(1: 1, w / w)$ are shown in Figure 3. PLGA RG502 and rivoceranib showed endothermic peaks at $38.5{ }^{\circ} \mathrm{C}$ and $194.5^{\circ} \mathrm{C}$, respectively (Figure 3A,B). It should be noted that the melting peak of rivoceranib appears in the polymer-drug physical mixture (Figure $3 \mathrm{C}$ ) and the polymer-drug blend films (Figure 3D-F) with minor shifts in position, suggesting the absence of significant interactions that affect the crystalline nature of rivoceranib. The minor changes in the peak position may be due to the blending process, which decreases the purity of each component and, therefore, and do not indicate the polymer-drug interactions [25]. The effect of polymer-drug interactions on the thermal behaviors of the components was also observed in the rivoceranib-loaded microspheres. As shown in the blend films, no significant changes in the thermograms of the microspheres were observed, although the endothermic peak for rivoceranib was not detectable, possibly due to the low quantity of the drug (Figure S1).

The potential polymer-drug interactions were further investigated using FTIR spectral analysis. The shifts of frequency and bandwidth of interacting groups indicate the changes in oscillation of the molecular dipoles in a polymer-drug mixture [26]. The characteristic peaks of PLGA ( $\mathrm{C}=\mathrm{O}$ at $1750-1705 \mathrm{~cm}^{-1}$; $\mathrm{OH}$ at $\left.3500-3400 \mathrm{~cm}^{-1}\right)$ and rivoceranib $\left(-\mathrm{COOH}\right.$ at $1750-1700 \mathrm{~cm}^{-1} ;-\mathrm{NH}$ at $700-600 \mathrm{~cm}^{-1} ;-\mathrm{C} \equiv \mathrm{N}$ at $2250-2200 \mathrm{~cm}^{-1}$ ) are shown in Figure 4A. The FTIR spectra of the polymer-drug physical blend films as well as the polymer-drug physical mixture did not show any absence of peaks of the functional groups in the spectra. In addition, no new bands were detected in the polymer-drug blend film. These results suggest that there are no significant chemical interactions, and the formation of new chemical linkages between PLGA and rivoceranib, demonstrating the significant compatibility between the two compounds. 


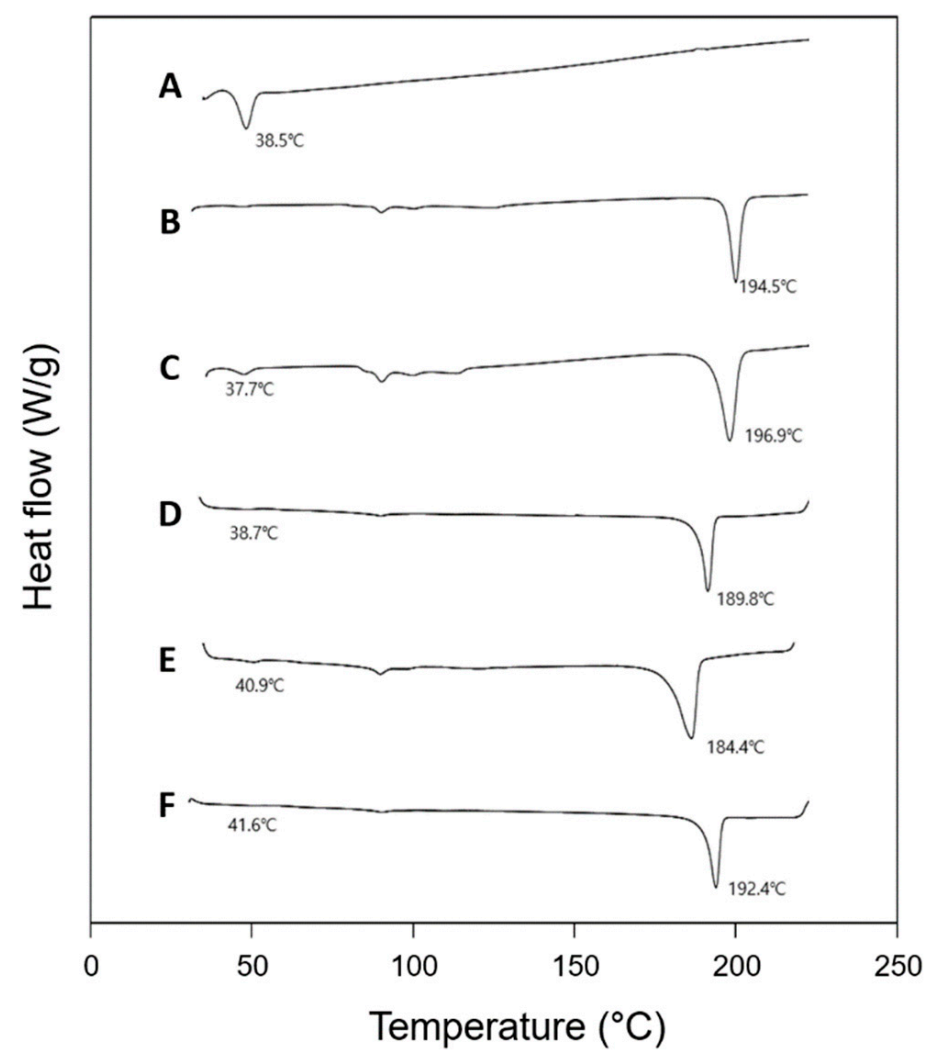

Figure 3. Differential scanning calorimetry (DSC) thermograms of (A) PLGA RG 502; (B) rivoceranib; (C) physical mixture (1:1) of PLGA RG502 and rivoceranib; (D) PLGA RG502 and rivoceranib (1:1) blend film; (E) PLGA RG502H and rivoceranib (1:1) blend film; (F) PLGA RG503H and rivoceranib (1:1) blend film. The downward peaks in the diagrams represent the endothermic behavior.

A

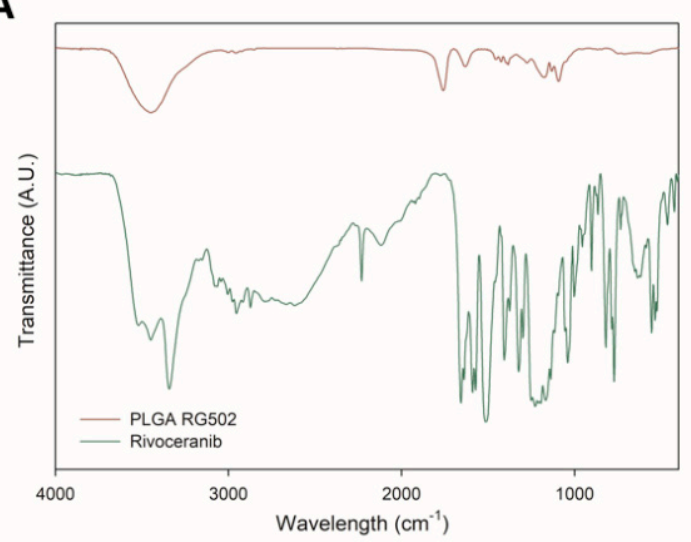

B

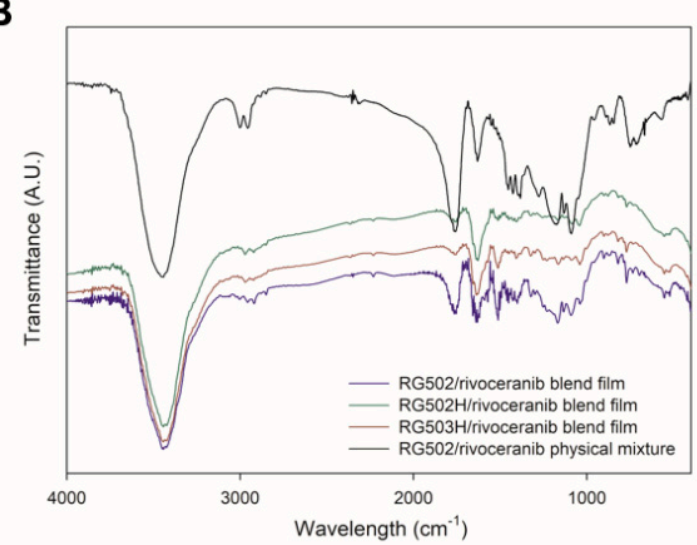

Figure 4. Fourier transform infrared (FTIR) spectrum of (A) PLGA RG502 polymer and rivoceranib; (B) PLGA RG502 and rivoceranib (1:1) blend film, PLGA RG502H and rivoceranib (1:1) blend film, PLGA RG503H and rivoceranib (1:1) blend film, and physical mixture (1:1) of PLGA RG502 and rivoceranib.

\subsection{Sustained Drug Release Profiles of Rivoceranib Microspheres}

In vitro rivoceranib release profiles from the rivoceranib microsphere formulations (Table 1) are shown in Figure 5. The release of free rivoceranib followed a zero-order kinetics with the cumulative drug release of $84 \%$ for 5 days. This result agrees with a previous observation with the release profile of a hydrophobic drug, paclitaxel, in an aqueous medium [27]. The microsphere formulations exhibited a burst release in a range from 1.4 to $5.7 \%$ over the initial $1 \mathrm{~h}$. After the initial burst, the microsphere formulations 
achieved sustained drug release for 50 days. The relatively low initial burst and slow drug release profiles would be due to the hydrophobic nature of rivoceranib. The RG502-2.0k and $\mathrm{RH} 502 \mathrm{H}-2.0 \mathrm{k}$ microspheres exhibited a similar drug release profile that resulted in 89.0 and $85.9 \%$ release during the experimental period, respectively. This indicated that the polar hydroxyl- and the ionizable carboxyl-end group of RG502 and RG502H did not significantly affect the drug release behaviors of the microspheres, suggesting the lack of significant interactions between the end group of the polymer chain and rivoceranib. The molecular weight of PLGA and the emulsification speed in the microsphere fabrication process significantly influence the release kinetics of the drug. The smaller microspheres prepared at a higher emulsification speed $(2000 \mathrm{rpm})$, including $502-2.0 \mathrm{k}, 502 \mathrm{H}-2.0 \mathrm{k}$, and $503 \mathrm{H}-2.0 \mathrm{k}$, exhibited much faster drug release profiles than ones prepared at a lower speed, owing to the increased surface area [28].

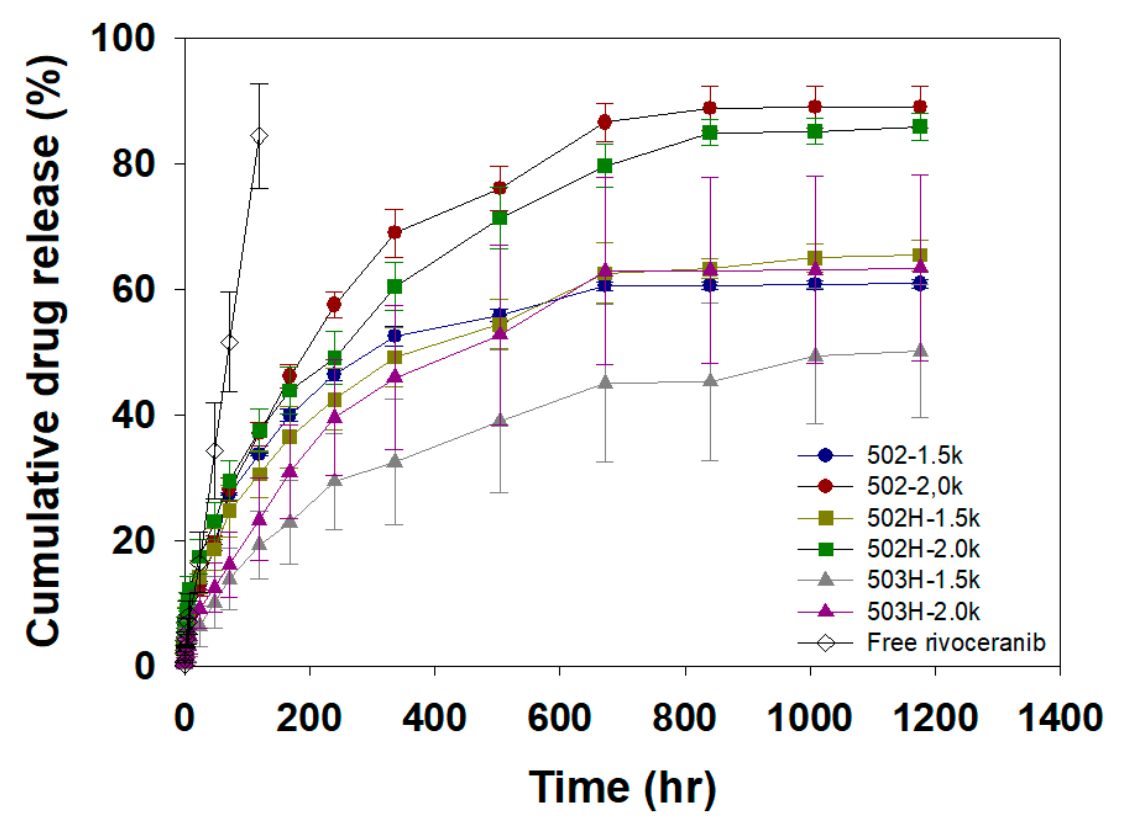

Figure 5. In vitro release curves of free rivoceranib and rivoceranib-loaded microspheres. The drug release profiles were represented as a mean $\pm \operatorname{SEM}(n=3)$.

\subsection{Suppression of CNV Formation in Mice by Intravitreal Administration of Rivoceranib Microspheres}

To evaluate the ability of rivoceranib microspheres to suppress CNV formation in vivo, a mouse with laser-induced CNV was used. This model induced a rupture of Bruch's membrane via laser photocoagulation, which causes the aberrant growth of new vessels from the choroid into the subretinal space [29]. The least effective dose of rivoceranib was determined as $1.0 \mu \mathrm{g}$, based on our previous results [30]. Immediately after laser photocoagulation, RG502H-2.0k microspheres containing $10 \mu \mathrm{g}$ rivoceranib, $\mathrm{RG} 502 \mathrm{H}-2.0 \mathrm{k}$ microspheres without the drug (blank microspheres), or PBS were intravitreally injected into mice. Two weeks later, the eyes were harvested and the area of $\mathrm{CNV}$ lesions was evaluated (Figure 6A). The posterior eyecups comprising retinal pigment epithelium (RPE), choroid, and sclera were flat-mounted and stained with isolectin B4 (IB4, stained in red). The CNV area was quantified by measuring the fluorescence intensity of images with isolectin B4-positive areas. Representative images of the IB4-stained CNV lesions show that the area of $\mathrm{CNV}$ lesions in $\mathrm{RG} 502 \mathrm{H}-2.0 \mathrm{k}$ microsphere-treated mice was much smaller than those in PBS- or blank microsphere-treated controls (Figure 6B). Quantitative analysis showed that the area of CNV lesions in mice treated with RG502H-2.0k microspheres was $2.78 \pm 0.21 \times 10^{3} \mu \mathrm{m}^{2}$, which was $2.12 \pm 0.27 \times 10^{3} \mu \mathrm{m}^{2}$ and $1.35 \pm 0.24 \times 10^{3} \mu \mathrm{m}^{2}$ lower than those in mice treated with blank microsphere and PBS, respectively. These 
results indicate that RG502H-2.0k microspheres efficiently block laser-induced pathological neovascularization in the choroid of mice.

A

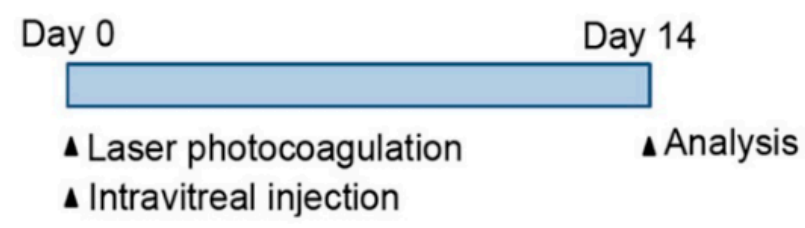

B

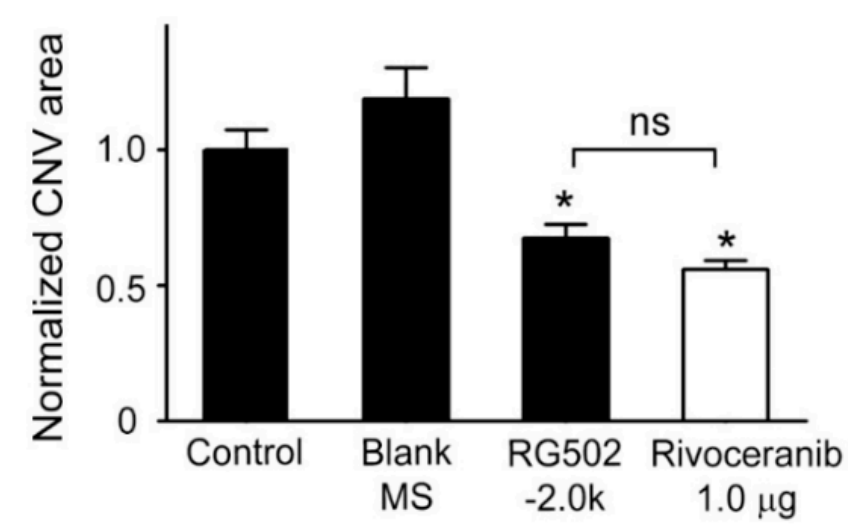

Figure 6. Inhibitory effect of rivoceranib-loaded PLGA microspheres and rivoceranib on laserinduced $\mathrm{CNV}$ formation in mice. (A) In vivo treatment schedule for the laser-induced choroidal neovascularization $(\mathrm{CNV})$ experiment. (B) Immediately after laser photocoagulation, the mice received a single intravitreal injection of PBS ( $1 \mu \mathrm{L} ; n=10$ mice, Control), drug free RG502H microspheres ( $10 \%$ microsphere in $1 \mu \mathrm{L}$ PBS, $n=4$ mice, Blank MS), and rivoceranib-loaded PLGA $502 \mathrm{H}$ microspheres (10 $\mu \mathrm{g}$ rivoceranib $/ 10 \% 502 \mathrm{H}-2.0 \mathrm{k}$ in $1 \mu \mathrm{L}$ PBS, $n=10$ mice). Two weeks later, the CNV area was analyzed. Rivoceranib (1.0 $\mu$ g rivoceranib in $1 \mu \mathrm{L}$ DMSO, $n=4$ mice) and PBS $(1 \mu \mathrm{L} ; n=10$ mice, Control). Two weeks later, the CNV area was analyzed. The CNV area was quantified by measuring the fluorescence intensity of images with isolectin IB4-positive areas. CNV areas of samples were expressed relative to that of control (one-way ANOVA with Bonferroni post hoc multiple comparison test, $\mathrm{ns}=$ not significant, ${ }^{*} p<0.05 \mathrm{vs}$. blank MS). Data are presented as the mean \pm SEM.

\section{Conclusions}

In wet-type age-related macular degeneration (AMD), VEGF and its receptors have been recognized as primary disease targets, in which the increased expression of VEGF contributes to aberrant angiogenesis and vascular leakage, leading to vision impairment and loss. In this regard, we investigated the possibility of repurposing an anticancer drug, rivoceranib, as a therapeutic intervention for AMD, and the effect of biodegradable microsphere-based sustained delivery of the drug on pathological angiogenesis in the eye using a laser-induced CNV model that is a common animal model of wet-type AMD. The rivoceranib microsphere achieved sustained in vitro drug release for 50 days. The intravitreal administration of the microsphere effectively suppressed the abnormal blood vessel formation of the foveal avascular zone (subfoveal CNV). Considering the biocompatibility and clinical significance of PLGA microspheres, the rivoceranib microspheres could be considered as an economical and effective alternative to antiangiogenic antibody therapy for AMD. 
Supplementary Materials: The following are available online at https://www.mdpi.com/article/10 .3390/pharmaceutics13101548/s1, Figure S1: Differential scanning calorimetry (DSC) thermograms of (A) PLGA RG502 microsphere (drug free); (B) PLGA RG502/rivoceranib microsphere (502-2.0k); (C) PLGA RG502H microsphere (drug free); (D) PLGA RG502H/rivoceranib microsphere (502H-2.0k); (E) PLGA RG503H microsphere (drug free); (F) PLGA RG503H/rivoceranib microsphere (503H-2.0k). The downward peaks in the diagrams represent the endothermic behavior.

Author Contributions: Conceptualization, J.H.J. and W.S.; methodology, E.S.K., M.S.L., H.J., S.Y.L., D.K. (Doha Kim), D.K. (Dahwun Kim), J.J., S.L., H.J.C. and D.M.K.; software, E.S.K., M.S.L. and H.J.; validation, E.S.K., M.S.L., J.J., S.L., H.J.C. and D.M.K.; formal analysis, E.S.K., M.S.L., H.J. and S.Y.L.; investigation, E.S.K., M.S.L., H.J., S.Y.L., D.K. (Doha Kim), D.K. (Dahwun Kim), J.J., S.L., H.J.C. and D.M.K.; resources, J.H.J.; data curation, E.S.K., M.S.L.; writing-original draft preparation, E.S.K. and M.S.L.; writing—review and editing, J.H.J.; supervision, J.H.J.; project administration, J.H.J.; funding acquisition, J.H.J. and W.S. All authors have read and agreed to the published version of the manuscript.

Funding: This research was supported by the National Research Foundation of Korea (NRF) grants (2018M3A9B502131913, 2020R1A4A4079931, 2020R1A2C2010449) funded by the Ministry of Science and ICT (MSIT).

Institutional Review Board Statement: The protocols were approved by the Institutional Animal Care and Use Committees (IACUC) of Chung-Ang University (IACUC number: 201800044, start date: 4 May 2018).

Informed Consent Statement: Not applicable.

Acknowledgments: This research was supported by the National Research Foundation of Korea (NRF) grants (2018M3A9B502131913, 2020R1A4A4079931, 2020R1A2C2010449) funded by the Ministry of Science and ICT (MSIT).

Conflicts of Interest: The authors declare no conflict of interest. The authors alone are responsible for the content and writing of this article.

\section{References}

1. Klaver, C.C.; Wolfs, R.C.; Vingerling, J.R.; Hofman, A.; de Jong, P.T. Age-specific prevalence and causes of blindness and visual impairment in an older population: The rotterdam study. Arch. Ophthalmol. 1998, 116, 653-658. [CrossRef] [PubMed]

2. $\quad$ Fleckenstein, M.; Keenan, T.D.; Guymer, R.H.; Chakravarthy, U.; Schmitz-Valckenberg, S.; Klaver, C.C.; Wong, W.T.; Chew, E.Y. Age-related macular degeneration. Nat. Rev. Dis. Primers 2021, 7, 31. [CrossRef]

3. Bird, A.C.; Bressler, N.M.; Bressler, S.B.; Chisholm, I.H.; Coscas, G.; Davis, M.D.; de Jong, P.T.; Klaver, C.C.; Klein, B.E.; Klein, R.; et al. An international classification and grading system for age-related maculopathy and age-related macular degeneration. The international arm epidemiological study group. Surv. Ophthalmol. 1995, 39, 367-374. [CrossRef]

4. Holz, F.G.; Wolfensberger, T.J.; Piguet, B.; Gross-Jendroska, M.; Wells, J.A.; Minassian, D.C.; Chisholm, I.H.; Bird, A.C. Bilateral macular drusen in age-related macular degeneration. Prognosis and risk factors. Ophthalmology 1994, 101, 1522-1528. [CrossRef]

5. Soubrane, G.; Bressler, N.M. Treatment of subfoveal choroidal neovascularisation in age related macular degeneration: Focus on clinical application of verteporfin photodynamic therapy. Br. J. Ophthalmol. 2001, 85, 483-495. [CrossRef]

6. Ferrara, N.; Gerber, H.P.; LeCouter, J. The biology of vegf and its receptors. Nat. Med. 2003, 9, 669-676. [CrossRef]

7. van Wijngaarden, P.; Coster, D.J.; Williams, K.A. Inhibitors of ocular neovascularization: Promises and potential problems. JAMA 2005, 293, 1509-1513. [CrossRef] [PubMed]

8. Jager, R.D.; Mieler, W.F.; Miller, J.W. Age-related macular degeneration. N. Engl. J. Med. 2008, 358, 2606-2617. [CrossRef] [PubMed]

9. Mandal, A.; Pal, D.; Agrahari, V.; Trinh, H.M.; Joseph, M.; Mitra, A.K. Ocular delivery of proteins and peptides: Challenges and novel formulation approaches. Adv. Drug Deliv. Rev. 2018, 126, 67-95. [CrossRef]

10. Wang, Y.; Wang, V.M.; Chan, C.C. The role of anti-inflammatory agents in age-related macular degeneration (amd) treatment. Eye 2011, 25, 127-139. [CrossRef]

11. Edelhauser, H.F.; Rowe-Rendleman, C.L.; Robinson, M.R.; Dawson, D.G.; Chader, G.J.; Grossniklaus, H.E.; Rittenhouse, K.D.; Wilson, C.G.; Weber, D.A.; Kuppermann, B.D.; et al. Ophthalmic drug delivery systems for the treatment of retinal diseases: Basic research to clinical applications. Investig. Ophth. Vis. Sci. 2010, 51, 5403-5420. [CrossRef]

12. Haller, J.A.; Bandello, F.; Belfort, R.; Blumenkranz, M.S.; Gillies, M.; Heier, J.; Loewenstein, A.; Yoon, Y.H.; Jiao, J.; Li, X.Y.; et al. Dexamethasone intravitreal implant in patients with macular edema related to branch or central retinal vein occlusion. Ophthalmology 2011, 118, 2453-2460. [CrossRef] 
13. Campochiaro, P.A.; Brown, D.M.; Pearson, A.; Chen, S.; Boyer, D.; Ruiz-Moreno, J.; Garretson, B.; Gupta, A.; Hariprasad, S.M.; Bailey, C.; et al. Sustained delivery fluocinolone acetonide vitreous inserts provide benefit for at least 3 years in patients with diabetic macular edema. Ophthalmology 2012, 119, 2125-2132. [CrossRef]

14. Herrero-Vanrell, R.; Bravo-Osuna, I.; Andres-Guerrero, V.; Vicario-de-la-Torre, M.; Molina-Martinez, I.T. The potential of using. Biodegradable microspheres in retinal diseases and other intraocular pathologies. Prog. Retin. Eye Res. 2014, 42, 27-43. [CrossRef]

15. Prajapati, V.D.; Jani, G.K.; Kapadia, J.R. Current knowledge on biodegradable microspheres in drug delivery. Expert Opin. Drug Del. 2015, 12, 1283-1299. [CrossRef] [PubMed]

16. Saishin, Y.; Silva, R.L.; Saishin, Y.; Callahan, K.; Schoch, C.; Ahlheim, M.; Lai, H.; Kane, F.; Brazzell, R.K.; Bodmer, D.; et al. Periocular injection of microspheres containing pkc412 inhibits choroidal neovascularization in a porcine model. Investig. Ophthalmol. Vis. Sci. 2003, 44, 4989-4993. [CrossRef]

17. Mietzner, R.; Kade, C.; Froemel, F.; Pauly, D.; Stamer, W.D.; Ohlmann, A.; Wegener, J.; Fuchshofer, R.; Breunig, M. Fasudil loaded plga microspheres as potential intravitreal depot formulation for glaucoma therapy. Pharmaceutics 2020, 12, 706. [CrossRef]

18. Carrasquillo, K.G.; Ricker, J.A.; Rigas, I.K.; Miller, J.W.; Gragoudas, E.S.; Adamis, A.P. Controlled delivery of the anti-vegf aptamer eye001 with poly(lactic-co-glycolic)acid microspheres. Investig. Ophthalmol. Vis. Sci. 2003, 44, 290-299. [CrossRef] [PubMed]

19. Olsson, A.K.; Dimberg, A.; Kreuger, J.; Claesson-Welsh, L. Vegf receptor signalling-in control of vascular function. Nat. Rev. Mol. Cell Biol. 2006, 7, 359-371. [CrossRef]

20. Kang, H.; Ho, A.L.; Muzaffar, J.; Bowles, D.W.; Kim, S.B.; Ahn, M.J.; Hanna, G.J.; Worden, F.P.; Yun, T.; Norton, S.; et al. A phase ii open-label, multicenter, study to evaluate the efficacy and safety of rivoceranib in subjects with recurrent or metastatic adenoid cystic carcinoma. J. Clin. Oncol. 2020, 38, TPS6597. [CrossRef]

21. Jeong, J.H.; Nguyen, H.K.; Lee, J.E.; Suh, W. Therapeutic effect of apatinib-loaded nanoparticles on diabetes-induced retinal vascular leakage. Int. J. Nanomed. 2016, 11, 3101-3109.

22. Lee, J.E.; Kim, K.L.; Kim, D.; Yeo, Y.; Han, H.; Kim, M.G.; Kim, S.H.; Kim, H.; Jeong, J.H.; Suh, W. A patinib-loaded nanoparticles suppress vascular endothelial growth factor-induced angiogenesis and experimental corneal neovascularization. Int. J. Nanomed. 2017, 12, 4813-4822. [CrossRef]

23. Kumar, N.; Goindi, S.; Saini, B.; Bansal, G. Thermal characterization and compatibility studies of itraconazole and excipients for development of solid lipid nanoparticles. J. Therm. Anal. Calorim. 2014, 115, 2375-2383. [CrossRef]

24. Mohamed, A.I.; Abd-Motagaly, A.M.; Ahmed, O.A.; Amin, S.; Ali, A.I. Investigation of drug-polymer compatibility using chemometric-assisted uv-spectrophotometry. Pharmaceutics 2017, 9, 7. [CrossRef]

25. Botha, S.A.; Lotter, A.P. Compatibility study between naproxen and tablet excipients using differential scanning calorimetry. Drug Dev. Ind. Pharm. 1990, 16, 673-683. [CrossRef]

26. Qiao, M.X.; Chen, D.W.; Ma, X.C.; Liu, Y.J. Injectable biodegradable temperature-responsive plga-peg-plga copolymers: Synthesis and effect of copolymer composition on the drug release from the copolymer-based hydrogels. Int. J. Pharm. 2005, 294, 103-112. [CrossRef]

27. Zhang, Z.; Wang, X.; Li, B.; Hou, Y.; Yang, J.; Yi, L. Development of a novel morphological paclitaxel-loaded plga microspheres for effective cancer therapy: In vitro and in vivo evaluations. Drug Deliv. 2018, 25, 166-177. [CrossRef]

28. Chen, W.; Palazzo, A.; Hennink, W.E.; Kok, R.J. Effect of particle size on drug loading and release kinetics of gefitinib-loaded plga microspheres. Mol. Pharm. 2017, 14, 459-467. [CrossRef]

29. Gong, Y.; Li, J.; Sun, Y.; Fu, Z.; Liu, C.H.; Evans, L.; Tian, K.; Saba, N.; Fredrick, T.; Morss, P.; et al. Optimization of an image-guided laser-induced choroidal neovascularization model in mice. PLoS ONE 2015, 10, e0132643. [CrossRef]

30. Kim, K.L.; Suh, W. Apatinib, an inhibitor of vascular endothelial growth factor receptor 2, suppresses pathologic ocular neovascularization in mice. Investig. Ophthalmol. Vis. Sci. 2017, 58, 3592-3599. [CrossRef] [PubMed] 